\title{
Nonlinear Coherent States of the Fokas-Lagerstrom Potential
}

\author{
M. Ashrafi ${ }^{1}$, A. Mahdifar ${ }^{2,3}$, E. Amooghorban ${ }^{1,4}$ \\ ${ }^{1}$ Department of Physics, Faculty of Basic Sciences, Shahrekord University, P.O. Box \\ 115, Shahrekord 88186-34141, Iran. \\ ${ }^{2}$ Department of Physics, Faculty of Science, University of Isfahan, Hezar Jerib, Isfahan, \\ 81746-73441, Iran \\ ${ }^{3}$ Quantum Optics Group, Department of Physics, Faculty of Science, University of \\ Isfahan, Hezar Jerib Str., Isfahan, 81746-73441, Iran \\ ${ }^{4}$ Nanotechnology Research Center, Shahrekord University, Shahrekord, 88186-34141, \\ Iran.
}

\begin{abstract}
In this paper, we introduce an algebraic approach to construct Fokas-Lagerstrom coherent states. To do so, we define deformed creation and annihilation operators associated to this system and investigate their algebra. We show that these operators satisfy the $f$-deformed Weyl-Heisenberg algebra. Then, we propose a theoretical scheme to generate the aforementioned coherent states. The present contribution shows that the Fokas-Lagerstrom nonlinear coherent states possess some non-classical features.

Keywords: Nonlinear Coherent States; Superintegrable Systems; Fokas-Lagerstrom Potential PACS: 03.65.Fd, 42.50.Dv
\end{abstract}

\section{Introduction}

It is well known that an $N$-dimensional classical or quantum system is called completely integrable if there are $N$ functionally independent well defined constants of motion (including the Hamiltonian) in involution [2, 32].

Email address: a.mahdifar@sci.ui.ac.ir (A. Mahdifar ${ }^{2,3}$ ) 
Furthermore, if it is possible to obtain $2 N-1$ constants of motion, the system is called maximally superintegrable, provided that the Poisson brackets (or the commutators) of these constants of motion with the Hamiltonian vanish (in general, only $N$ of $2 N-1$ constants are in involution) [32, 23, 5, 6, 33, 4, 28]. Famous examples of the superintegrable systems are the isotropic harmonic oscillator and the Kepler-Coulomb potential. It is worthwhile to note that their higher order symmetries, play the fundamental role in solvability and other interesting properties of these physical systems.

In the last decades, coherent states of the harmonic oscillator [8] and generalized coherent states associated with various algebras [22, 11, 1] have been playing an important role in various branches of physics. The coherent states, which defined as the right eigenstates of the annihilation operator $\hat{a}$, are the quantum states with classical-like properties. In other words, they are the closest analogue to classical states. On the other hand, the generalized coherent states exhibit some nonclassical properties and, therefore, have received an ever-increasing interest during the last decades. Among the generalized coherent states, the nonlinear coherent states or f-deformed coherent states [26, 24, 25] have attracted many interests in recent years due to their applications in quantum optics and quantum technology. It is shown that the statistical characteristics of these states exhibit some nonclassical features, such as photon antibunching [10], sub-Poissonian photon statistics [29] and squeezing [9]. These states, which are associated with nonlinear algebras [26, 18], could be generated in the center-of-mass motion of an appropriately laser-driven trapped ion [30, 15] and in a micromaser under intensity-dependent atom-field interaction [20].

Recently, one of us defined the nonlinear coherent states for some of the two-dimensional superintegrable systems, including the isotropic harmonic oscillator on a sphere [16] and the Kepler-Coulomb problem on a sphere [14]. In the present contribution, we study the Fokas-Lagerstrom potential as the another example of the two-dimensional superintegrable systems [7]. Our approach which is based on the f-deformed harmonic oscillator algebra and the nonlinear coherent states, can increase the insight about the FokasLagerstrom system. Of course, the Fokas-Lagerstrom potential was considered previously [7, 3]. The distinction between the present paper and the mentioned references is that our approach is based on the algebraic methods of the nonlinear coherent states.

The paper is organized as follows. In Sec. 2, we briefly review the superintegrable systems. By using the nonlinear oscillator approach in Sec. 3, we 
investigate the Fokas-Lagerstrom system, as an example of two-dimensional superintegrable systems and show that the algebra of the this system can be considered as an $f$-deformed Weyl-Heisenberg algebra. We define nonlinear coherent states for the Fokas-Lagerstrom potential and examine their resolution of identity in Sec. 4. In Sec. 5, we propose a scheme to generate the aforementioned coherent states. Sec. 6] is devoted to the study of the quantum statistical properties of the constructed nonlinear coherent states, including mean number of photons, Mandel parameter and quadrature squeezing. Finally, the summary and concluding remarks are given in Sec. 7 .

\section{Superintegrable systems}

In classical mechanics, an $N$-dimensional system is called superintegrable, if it has more than $N$ independent constants of motion. Furthermore, if the system has exactly $(2 N-1)$ independent constants of motion (the maximum number) and also if all of these constants are single valued and globally defined, then the system is called maximally superintegrable system [2, 32].

To be specific, for a classical system in two dimensions, described by the following Hamiltonian:

$$
H=H\left(x, y, p_{x}, p_{y}\right),
$$

if there exist two independent constants of motion $C_{1}$ and $C_{2}$, so that we have,

$$
\begin{aligned}
& \left\{H, C_{1}\right\}_{P B}=\left\{H, C_{2}\right\}_{P B}=0, \\
& \left\{C_{1}, C_{2}\right\}_{P B} \neq 0,
\end{aligned}
$$

then this system is called a superintegrable system. Here, $\{,\}_{P B}$ denotes the Poisson bracket [32].

In quantum mechanics, a two-dimensional system described by a Hamiltonian $\hat{H}$ is called integrable, if is possible to find an operator $\hat{C}_{1}$ commuting with the $\hat{H}$ [32]:

$$
\left[\hat{H}, \hat{C}_{1}\right]=0 \text {. }
$$

This system is also called superintegrable, if there exists another operator $\hat{C}_{2}$, linearly independent of $\hat{H}$ and $\hat{C}_{1}$, that commute with $\hat{H}$ but not commute with $\hat{C}_{1}$, so that,

$$
\left[\hat{H}, \hat{C}_{2}\right]=0,\left[\hat{C}_{1}, \hat{C}_{2}\right] \neq 0
$$




\section{Fokas- Lagerstrom system}

We consider the quantum Fokas-Lagerstrom system, described by the Hamiltonian [7]:

$$
\hat{H}=\frac{1}{2}\left(\hat{P}_{x}^{2}+\hat{P}_{y}^{2}\right)+\frac{\hat{x}^{2}}{2}+\frac{\hat{y}^{2}}{18}
$$

(in this paper we put $\hbar=m=\omega=1$ ). By introducing the following operators:

$$
\begin{aligned}
\hat{J} & =\hat{P}_{x}^{2}+\hat{x}^{2}, \\
\hat{B} & =\frac{1}{2}\left\{\hat{X} \hat{P}_{y}-\hat{Y} \hat{P}_{x}, \hat{P}_{y}^{2}\right\}+\frac{\hat{Y}^{3} \hat{P}_{x}}{27}-\frac{\left\{\hat{X} \hat{Y}^{2}, \hat{P}_{y}\right\}}{6},
\end{aligned}
$$

where $\{$,$\} is the usual anticommutator, and making use of Eq.(5), it can be$ easily shown that the following commutation relations hold [3],

$$
\begin{aligned}
{[\hat{H}, \hat{B}] } & =[\hat{H}, \hat{J}]=0, \\
{[\hat{J}, \hat{R}] } & =4 \hat{B} \\
{[\hat{J}, \hat{B}] } & =\hat{R} .
\end{aligned}
$$

With these in mind, and considering the definition of the superintegrable systems, it is clear that the Fokas-Lagerstrom system is a quantum superintegrable system. Now, by defining the following operators:

$$
\begin{aligned}
\hat{n} & =\frac{\hat{J}}{2}-u \hat{I}, \\
\hat{A}^{\dagger} & =\hat{B}+\frac{\hat{R}}{2}, \\
\hat{A} & =\hat{B}-\frac{\hat{R}}{2},
\end{aligned}
$$

where $u$ is a constant to be determined, it is seen that the operators $\hat{A}, \hat{A}^{\dagger}$ and $\hat{n}$ satisfy the following closed algebra [3]:

$$
\begin{aligned}
{\left[\hat{n}, \hat{A}^{\dagger}\right] } & =\hat{A}^{\dagger} \\
{[\hat{n}, \hat{A}] } & =-\hat{A} \\
{\left[\hat{A}, \hat{A}^{\dagger}\right] } & =\Phi(\hat{H}, \hat{n}+1)-\Phi(\hat{H}, \hat{n}) .
\end{aligned}
$$


where the structure function $\phi$ is given by

$$
\begin{aligned}
\Phi(E, x)= & \frac{1}{9}(2 x+2 u-1)(2 E+1-2 x-2 u) \\
& \times(6 E-6 u-6 x)(6 E-6 u+5-6 x) .
\end{aligned}
$$

This structure function, for $n \geq 0$ is a real definite positive function and also $\phi(E, 0)=0$. Now, for each energy eigenvalue it is possible to define the corresponding Fock space as below [3, 21],

$$
\begin{aligned}
\hat{H}|E, n\rangle & =E|E, n\rangle, \\
\hat{N}|E, n\rangle & =n|E, n\rangle, \\
\hat{A}|E, 0\rangle & =0 \\
|E, n\rangle & =\frac{\left(A^{\dagger}\right)^{n}}{\sqrt{[\phi(E, n)] !}}|E, 0\rangle .
\end{aligned}
$$

For a discrete energy eigenvalue $E$, there is the $N+1$-dimensional degeneracy. So, we deal with an $N$-dimensional Fock space corresponding to that eigenvalue energy as,

$$
\begin{aligned}
\hat{H}|N, n\rangle & =E_{N}|N, n\rangle, & & N=0,1,2, \ldots \\
\hat{n}|N, n\rangle & =n|N, n\rangle & & n=0,1,2, \ldots, N
\end{aligned}
$$

The combination of this restriction along with $\phi(E, 0)=0$ and $\phi(E, N+1)=$ 0 , determines $u$ and the possible energy eigenvalues as below [3]:

$$
\begin{aligned}
u & =\frac{1}{2}, \\
E_{N} & =N+1, \\
E_{N} & =N+\frac{2}{3}, \\
E_{N} & =N+\frac{4}{3} .
\end{aligned}
$$

Therefore, the allowable structure functions are respectively given by,

$$
\Phi\left(E_{N}, x\right)=16 x(N+1-x)(N+A-x)(N+B-x),
$$

where, the constants $A$ and $B$ are corresponding to one of the pairs $(2 / 3,4 / 3)$, $(2 / 3,1 / 3)$ or $(5 / 3,4 / 3)$. 
As is well known, the $f$-deformed annihilation and creation operators associated with an $f$-deformed harmonic oscillator can be defined as [18],

$$
\begin{aligned}
\hat{A} & =\hat{a} f(\hat{n})=f(\hat{n}+1) \hat{a} \\
\hat{A}^{\dagger} & =f^{\dagger}(\hat{n}) \hat{a}^{\dagger}=\hat{a}^{\dagger} f^{\dagger}(\hat{n}+1),
\end{aligned}
$$

where $\hat{a}$, $\hat{a}^{\dagger}$ and $\hat{n}$ are the bosonic annihilation, creation and number operators, respectively, and $f(\hat{n})$ is a real nonnegative deformation function. These deformed operators satisfy the commutation relation

$$
\left[\hat{A}, \hat{A}^{\dagger}\right]=(\hat{n}+1) f(\hat{n}+1) \hat{f}^{\dagger}(\hat{n}+1)-\hat{n} f(\hat{n}) f^{\dagger}(\hat{n}) .
$$

On the other hand, with respect to the algebraic structure of Fokas-Lagerstrom superintegrable systems, Eq. (10), we have

$$
\left[\hat{A}, \hat{A}^{\dagger}\right]=\Phi(\hat{H}, N+1)-\Phi(\hat{H}, N) .
$$

Now, if we deal with a constant energy, $E_{N}$, then $\Phi(\hat{H}, \hat{n})$ depends only on $\hat{n}$ and if we now compare Eq. (21) with Eq. (22), we find that:

$$
n f^{2}(n)=\Phi\left(E_{N}, n\right) .
$$

Therefore, we can consider the algebra of Fokas-Lagerstrom system as a deformed Weyl-Heisenberg algebra with the following deformation function:

$$
f(n)=\sqrt{16(N+1-n)(N+A-n)(N+B-n)} .
$$

Now, by using the deformed creation and annihilation operators, $A$ and $A^{\dagger}$, we arrive at

$$
\hat{A}|0\rangle=0=\hat{A}^{\dagger}|N\rangle
$$

Thus, we conclude that for any constant $N$, corresponding to the constant value of energy $E_{N}$, there is a Hilbert space with finite dimension.

In the next section, we intend to construct the finite-dimensional coherent states corresponding to the Fokas-Lagrestrom potential.

\section{Fokas-Lagrstrom nonlinear coherent states}

Let us now turn to define the finite-dimensional nonlinear coherent states corresponding to the Fokas-Lagrstrom potential. We follow the formalism of 
truncated coherent state approach introduced in [13], to define the FokasLagrstrom nonlinear coherent states (FLNCSs). Therefore, we have:

$$
|z\rangle_{F . L}=C^{-\frac{1}{2}}\left(|z|^{2}\right) \exp \left(z \hat{A}^{\dagger}\right)|0\rangle
$$

where $z$ is a complex number. After some calculations, the above nonlinear coherent state can be recast into the following form:

$$
|z\rangle_{F . L}=C^{-\frac{1}{2}}\left(|z|^{2}\right) \sum_{n=0}^{N} \frac{z^{n}}{\sqrt{\rho(n)}}|n\rangle
$$

where $\rho(n)$ is defined as:

$$
\begin{aligned}
\rho(n)= & \left(\frac{1}{16}\right)^{n}\left[\frac{\Gamma(n+1) \Gamma(N-n+1)}{N !}\right]\left[\frac{\Gamma(N+A-n)}{\Gamma(N+A)}\right] \\
& \times\left[\frac{\Gamma(N+B-n)}{\Gamma(N+B)}\right] .
\end{aligned}
$$

Here, $\Gamma$ is the gamma function, and the normalization constant $C$ is given by,

$$
C\left(|z|^{2}\right)=\sum_{n=0}^{N} \frac{|z|^{2 n}}{\rho(n)}
$$

\subsection{Resolution of identity}

In this section, we intend to show that the constructed FLNCSs form an overcomplete set. In other words, we would show:

$$
\int d^{2} z|z\rangle W\left(|z|^{2}\right)\left\langle z\left|=\sum_{n=0}^{N}\right| n\right\rangle\langle n|=\mathbb{I} .
$$

The resolution of identity can be achieved by finding a measure function $W\left(|z|^{2}\right)$. For this purpose, by substituting $|z\rangle_{F . L}$ from the Eq. (27) into Eq. (30) we obtain,

$$
\int d^{2} z|z\rangle_{F . L} W\left(|z|^{2}\right)_{F . L}\left\langle\left. z\left|=\pi \sum_{n=0}^{\infty} \frac{|n\rangle\langle n|}{\rho(n)} \int_{0}^{\infty} d\left(|z|^{2}\right)\right| z\right|^{2 n} \frac{W\left(|z|^{2}\right)}{C\left(|z|^{2}\right)}\right.
$$


where we have used: $z=|z| e^{i \theta}$ and $d^{2} z=\frac{1}{2} d\left(|z|^{2}\right) d \theta$. Now, by using the change of variable $x=|z|^{2}$ and considering $w(x)=\pi \frac{W\left(|z|^{2}\right)}{C\left(|z|^{2}\right)}$, we should have:

$$
\int_{0}^{\infty} d x x^{n} w(x)=\rho(n) .
$$

The above integral is called the moment problem and well-known mathematical methods such as Mellin transformations can be used to solve it [12, 19]. From definition the of Meijers $G$-function, it follows that,

$$
\begin{array}{r}
d x x^{k-1} G_{p, q}^{m, n}\left(\beta x \mid \begin{array}{l}
a_{1}, \cdots, a_{n}, a_{n+1}, \cdots, a_{p} \\
b_{1}, \cdots, b_{m}, b_{m+1}, \cdots, b_{q}
\end{array}\right) \\
=\frac{1}{\beta^{k}} \frac{\prod_{j=1}^{m} \Gamma\left(b_{j}+k\right) \prod_{j=1}^{n} \Gamma\left(1-a_{j}-k\right)}{\prod_{j=m+1}^{q} \Gamma\left(1-b_{j}-k\right) \prod_{j=n+1}^{p} \Gamma\left(a_{j}+k\right)} .
\end{array}
$$

Therefore, by comparing Eqs. (32) and (33), we find that the weight function can be written as,

$$
\begin{aligned}
w(x)= & \frac{16}{N ! \Gamma(N+A) \Gamma(N+B)} \\
& \times G_{3,1}^{1,3}(16 x \mid-N-1,-N-A,-N-B) .
\end{aligned}
$$

In this manner, it is seen that the FLNCSs satisfy the resolution of identity and consequently form an overcomplete set.

\section{The Physical Generation of FLNCSs}

To generate an arbitrary but finite superposition of Fock states, a physical scheme has been proposed in Ref. [31]. In this method, $\mathrm{N}$ two-level atoms which are prepared in a specific superposition of the excited state $|a\rangle$ and the ground state $|b\rangle$ interact with a resonant mode of radiation field in a cavity via the Jaynes-Cummings Hamiltonian. The cavity field is initially in the vacuum state. By choosing atoms with appropriate initial states, it is possible to produce the desired state. In the resonator, the measurement of the internal state of the atom after it has passed through the cavity leaves the quantum states of field in a pure state. After the interaction of $(K-1)^{\text {'t }}$ th atom with cavity field, we make a measurement on the atomic state. If we 
find the atom in the ground state $|b\rangle$, the state of the radiation field reads:

$$
\left|\varphi^{(k-1)}\right\rangle=\sum_{n} \varphi_{n}^{(k-1)}|n\rangle
$$

If the atom found in the excited state, our attempt to create the desired field state fails and we go back to vacuum state and start the procedure again. Therefore, the state of the atom-field system after the $K^{6}$ th atom in the atomic state $|a\rangle+i \varepsilon_{k}|b\rangle$ exit the cavity is given by

$$
\begin{aligned}
\left|\Phi^{(k)}\right\rangle= & \sum_{n} \varphi_{n}^{(k-1)} C_{n}^{(k)}|n, a\rangle-i S_{n}^{(k)}|n+1, b\rangle \\
& +i \epsilon_{k} C_{n-1}^{(k)}|n, b\rangle+\epsilon_{k} S_{n-1}^{(k)}|n-1, a\rangle,
\end{aligned}
$$

where

$$
\begin{aligned}
C_{n}^{k} & =\cos \left(g \tau_{k} \sqrt{n+1}\right), \\
S_{n}^{k} & =\sin \left(g \tau_{k} \sqrt{n+1}\right) .
\end{aligned}
$$

Besides, the new coefficients $\varphi_{n}{ }^{(k)}$ are given in terms of the $\varphi_{n}{ }^{(k-1)}$ as

$$
\varphi_{n}^{(k)}=S_{n-1}^{(k)} \varphi_{n-1}^{(k-1)}-\varepsilon_{k} C_{n-1}^{(k)} \varphi_{n}^{(k-1)} .
$$

We are now in a position to generate the finite-dimensional FLNCSs $|z\rangle_{F . L}$ based on this approach. For this purpose, we need to obtain that field combination state,

$$
\left|\varphi^{(N-1)}\right\rangle=\sum_{n=0}^{N-1} \varphi_{n}^{(N-1)}|n\rangle,
$$

of $N$ number states such that after the $N^{6}$ th atom with the atomic superposition $|a\rangle+i \epsilon_{N}|b\rangle$ passed through the cavity and has been detected in the ground state, the field in the cavity become,

$$
|z\rangle_{F . L}=\sum_{n=0}^{N} d_{n}|n\rangle,
$$

where

$$
d_{n}=C^{-\frac{1}{2}}\left(|z|^{2}\right) \frac{z^{n}}{\sqrt{\rho(n)}} .
$$


By using Eq. (39), we get a set of $N+1$ equations which $N$ coefficients $\varphi_{n}{ }^{(N-1)}$ and the parameter $\varepsilon_{N}$ can be obtained from it. The unknown coefficient $\varphi_{n}^{(N-1)}$ is given by [31]

$$
\varphi_{n}^{(N-1)}=\sum_{v=1}^{N-n}\left[\prod_{\mu=n}^{n+\mu-2} \frac{C_{\mu}^{(N)}}{S_{\mu}^{(N)}}\right] \frac{d_{n+v}}{S_{n+v-1}^{(N)}} \varepsilon_{N}^{v-1} .
$$

We also have the characteristic equation for $\varepsilon_{N}$ as

$$
d_{0}+\sum_{v=1}^{N}\left[\prod_{\mu=0}^{v-2} \frac{C_{\mu}^{(N)}}{S_{\mu}^{(N)}}\right] \frac{d_{v}}{S_{v-1}^{(N)}} \varepsilon_{N}^{v}=0
$$

We choose $\varepsilon_{N}$ as one of the $N$ roots of the Eq. (44) which is a polynomial of degree $N$. In order to obtain other parameters $\varepsilon_{N-1}, \ldots, \varepsilon_{2}$ and $\varepsilon_{1}$ by a recurrence relation, we take $\left|\varphi^{(N-1)}\right\rangle$ as a new desired state which can be generated by sending $N-1$ atoms through the cavity. By following the same procedure, $N-1$ coefficient $\varphi^{(N-2)}$ and the parameter $\varepsilon_{N-1}$ are obtained. Repeating the calculations yields a sequence of complex numbers $\varepsilon_{1}, \varepsilon_{2}, \ldots, \varepsilon_{N}$ that defines the internal state of the $N$ injected atoms into the cavity in order to generate the desired state $|z\rangle_{F L}$.

To be specific, we illustrate step by step generation of the FLNCSs with $N=2$ by using the space distribution $Q$-function [27]. The $Q$-function of a pure state $|\psi\rangle$ is defined as

$$
Q(\alpha)=\frac{|\langle\alpha \mid \psi\rangle|^{2}}{\pi}
$$

where $|\alpha\rangle$ is the standard coherent state.

Fig. 1 displays the $Q$-function and the counter lines of the $Q$-function for the cavity state $\left|\varphi^{(k)}\right\rangle$ after the $k^{6}$ th atom has passed through the resonator

and has been detected in ground state for a fix interaction parameter $g \tau=\frac{\pi}{5}$ and $k=0,1,2$.

\section{Quantum Statistical Properties of the FLNCSs}

In this section, we we shall proceed to study some quantum statistical properties of the FLNCSs, including probability of finding $n$ quanta, mean number of photons, Mandel parameter and quadrature squeezing. 


\subsection{Photons-number distribution}

By using Eq. (27), the probability $P(n)$ of finding $n$ photon in the FLNCSs is given by

$$
P(n)=\frac{1}{\sum_{n=0}^{N} \frac{|z|^{2 n}}{\rho(n)}} \frac{|z|^{2 n}}{\rho(n)} .
$$

As it is clear from the above complex equation, it is difficult to predict the results analytically. Therefore, in Fig. 2 we show the effect of the parameter $z$ on the probability of finding $n$ photons in the FLNCSs with $A=2 / 3$ and $B=1 / 3$. To get further insight, let us consider the limiting case $z \longrightarrow \infty$. Form Eq. (46), we obtain that the probabilities $P(n)$ tends to $\delta_{n, N}$. In other words, by increasing $z$, the FLNCS $|z\rangle$ approaches to the number state $|N\rangle$.

The mean number of photons in the FLNCSs is calculated as follows:

$$
\langle\hat{n}\rangle={ }_{F . L}\left\langle z\left|\hat{a}^{\dagger} \hat{a}\right| z\right\rangle_{F . L}=\sum_{n=0}^{N} n P(n) .
$$

In Fig. 3, the mean number of photons in FLNCSs is plotted in terms of $z$ for $(A, B)=(2 / 3,1 / 3)$ and for different values of $N$. It is seen that for a constant $N$, by increasing $z$, the mean number of photons increases, and in the limit of $z \longrightarrow \infty$ we get: ${ }_{F . L}\langle z|\hat{n}| z\rangle_{F . L} \rightarrow N$. In addition, for a fixed value of $z$, the mean number of photons is increased by the increasing the dimension of Hilbert space $N$. It is worth noting that these results are consistent with the results in Fig. 2 .

\subsection{Mandel parameter}

In this subsection, we investigate deviation from the Poisson distribution for the FLNCSs by using the Mandel parameter. This parameter is defined as [17]:

$$
M=\frac{(\Delta n)^{2}-\langle n\rangle}{\langle n\rangle},
$$

where the positive, zero and negative values represent super-Poissonian, Poissonian and sub-Poissonian distribution, respectively. Due to the complexity of the final form of the Mandel parameter for the FLNCSs, we do not attempt to obtain its analytic form. Instead, we numerically study the Mandel parameter for these states. In Fig. 4, we have plotted the Mandel parameter of the FLNCSs with respect to $z$ for $(A, B)=(2 / 3,1 / 3)$ and different values 
of $N$. The results show that, for a fixed value of $N$, the photon counting statistic of the FLNCSs becomes more sub-Poissonian with increasing $z$ and then tends to -1 at very large values of $z$. We can justify this result by the fact that the quantum number state $|N\rangle$ has the Mandel parameter $M=-1$; since, the FLNCS approaches to the state $|N\rangle$ with increasing $z$, as is seen in Fig. 2,

\subsection{Quadrature squeezing}

In this subsection, we consider the quadrature operators $\hat{X}_{1}$ and $\hat{X}_{2}$ defined in terms of creation and annihilation operators $\hat{a}$ and $\hat{a}^{\dagger}$ as follows :

$$
\begin{aligned}
& \hat{X}_{1}=\frac{1}{2}\left(\hat{a} e^{i \phi}+\hat{a}^{\dagger} e^{-i \phi}\right), \\
& \hat{X}_{2}=\frac{1}{2 i}\left(\hat{a} e^{i \phi}-\hat{a}^{\dagger} e^{-i \phi}\right) .
\end{aligned}
$$

By using the commutation relation of $\hat{a}$ and $\hat{a}^{\dagger}$, the following uncertainty relation is obtained

$$
\left(\Delta X_{1}\right)^{2}\left(\Delta X_{2}\right)^{2} \geq \frac{1}{16}\left|\left\langle\left[\hat{X}_{1}, \hat{X}_{2}\right]\right\rangle\right|^{2}=\frac{1}{16} .
$$

As is known, the quadrature squeezing occurs if we have $\left(\Delta X_{i}\right)^{2}<1 / 4(i=$ 1or2) or equally $S_{i} \equiv 4\left(\Delta X_{i}\right)^{2}-1<0$. Figs. 5(a) and 5(b) display the squeezing parameters $S_{1}$ and $S_{2}$, respectively, for the FLNCSs with $(A, B)=$ $(2 / 3,1 / 3)$ as a function of $\varphi$ for $z=0.02$ and different values of $N$.

It is seen that the range of the parameter $\phi$, in which the squeezing occurs, decreases by increasing $N$. In this range, the effect of increasing the dimension $N$ on the squeezing is dependant on $\phi$. Besides, in the areas where the maximum squeezing occurs, an increase in $N$ leads to increase the squeezing, while for other $\phi$ 's available in this range, increasing of $N$ causes a reduction in the squeezing.

\section{Summary and Concluding Remarks}

In this paper, we have introduced an algebraic approach to the FokasLagerstrom system. We have found that the two-dimensional Fokas-Lagerstrom algebra can be considered as a deformed one-dimensional harmonic oscillator algebra. In this manner, we have succeeded to construct the nonlinear coherent states for this potential and studied their quantum statistical properties. Finally, we have proposed a physical scheme to generate the FLNCSs. 


\section{References}

[1] Ali S T, Antoine J-P and Gazeau J-P Coherent States, Wavelets and Their Generalizations (New York: Springer, 2000)

[2] Arnold V I Mathematical Methods of Classical Mechanics, Graduate Texts in Mathematics (Berlin: Springer-Verlag, 1978).

[3] Bonatsos D, Daskaloyannis C and Kokkotas K Phys. Rev. A 50 (1994) 3700

[4] Eisenhart L P Ann. Math. 35 (1934) 287.

[5] Evans N W J. Math. Phys. 32 (1991) 3369.

[6] Evans N W Phys. Lett. A 147 (1990) 483.

[7] Fokas A S and Lagerstrom P A J. Math. Anal. AppL 74 (1980) 325

[8] Glauber R J Phys. Rev. 136 (1963) 2529; Glauber R J Phys. Rev. 131 (1963) 2766; Glauber R J Phys. Rev. Lett. 10 (1963) 84

[9] Hong C K and Mandel L Phys. Rev. Lett. 54 (1985) 323; Wu L A, Kimble H J, Hall J L and Wu H Phys. Rev. Lett. 57 (1986) 2520

[10] Kimble H J, Dagenais M and Mandel L Phys. Rev. Lett. 39 (1977) 691

[11] Klauder J R and Skagerstam B S Coherent States, Applications in Physics and Mathematical Physics (Singapore: World Scientific, 1985)

[12] Klauder J R, Peson K A and Sixdeniers J M Phys Rev. A, 64 (2001) 013817

[13] Kuang L M, Wang F B and Zhou Y G Phys. Lett. A 183 (1993) 1

[14] Mahdifar A, Hoseinzadeh T, Bagheri Harouni M Ann Phys. 335 (2015) 21

[15] Mahdifar A, Vogel W, Richter Th, Roknizadeh R and Naderi M H Phys. Rev. A 78 (2008) 063814.

[16] Mahdifar A, Roknizadeh R and Naderi M H J. Phys. A 39 (2006) 7003 
[17] Mandel L and Wolf E Optical Coherence and Quantum Optics (Cambridge: Cambridge University Press, 1995)

[18] Manko V I, Marmo G, Sudarshan E C G and Zaccaria F Phys. Scr. 55 (1997) 528

[19] Mathai A M and Saxena R K Generalized Hypergeometric Functions with Applications in Statistics and Physical Sciences (New York: Springer-Verlag, 1973)

[20] Naderi M H, Soltanolkotabi M and Roknizadeh R Eur. Phys. J. D. 32 (2005) 397.

[21] Panahi H and Alizadeh Z, Chin. Phys. B 22 (2013) 060304.

[22] Perelomov A P Generalized Coherent States and Their Applications (Berlin: Springer, 1986)

[23] Ranada M F J. Math. Phys. 38 (1997) 4165.

[24] Roknizadeh R and Tavassoly M K J. Phys. A: Math. Gen. 37 (2004) 8111.

[25] Roknizadeh R and Tavassoly M K J. Math. Phys. 46(4) (2005) 042110.

[26] Solomon A I Phys. Lett. A 196 (1994) 29; Katriel J and Solomon A I Phys. Rev. A 49 (1994) 5149; Shanta P, Chaturvdi S, Srinivasan V and Jagannathan R J. Phys. A: Math. Gen. 27 (1994) 6433

[27] Scully M O and Zubairy M S Quantum Optics (Cambridge: Cambridge University Press, 1997)

[28] Tegment A and Vercin A Int. J. Mod. Phys. A 19 (2004) 393.

[29] Teich M C and Saleh B E A J. Opt. Soc. Am. B 2 (1985) 275

[30] Vogel W and de Matos Filho R L Phys. Rev. A 54 (1996) 4560

[31] Vogel K, Akulin V M and Schleich W P Phys. Rev. Lett 71 (1993) 1816

[32] Winternitz P Superintegrable systems in classical and quantum mechanics, in: New trends in integrability and partial solvability 281, 2004 (Dordrecht: Kluwer) 
[33] Wojciechowski S Phys. Lett. A 95 (1983) 279. 

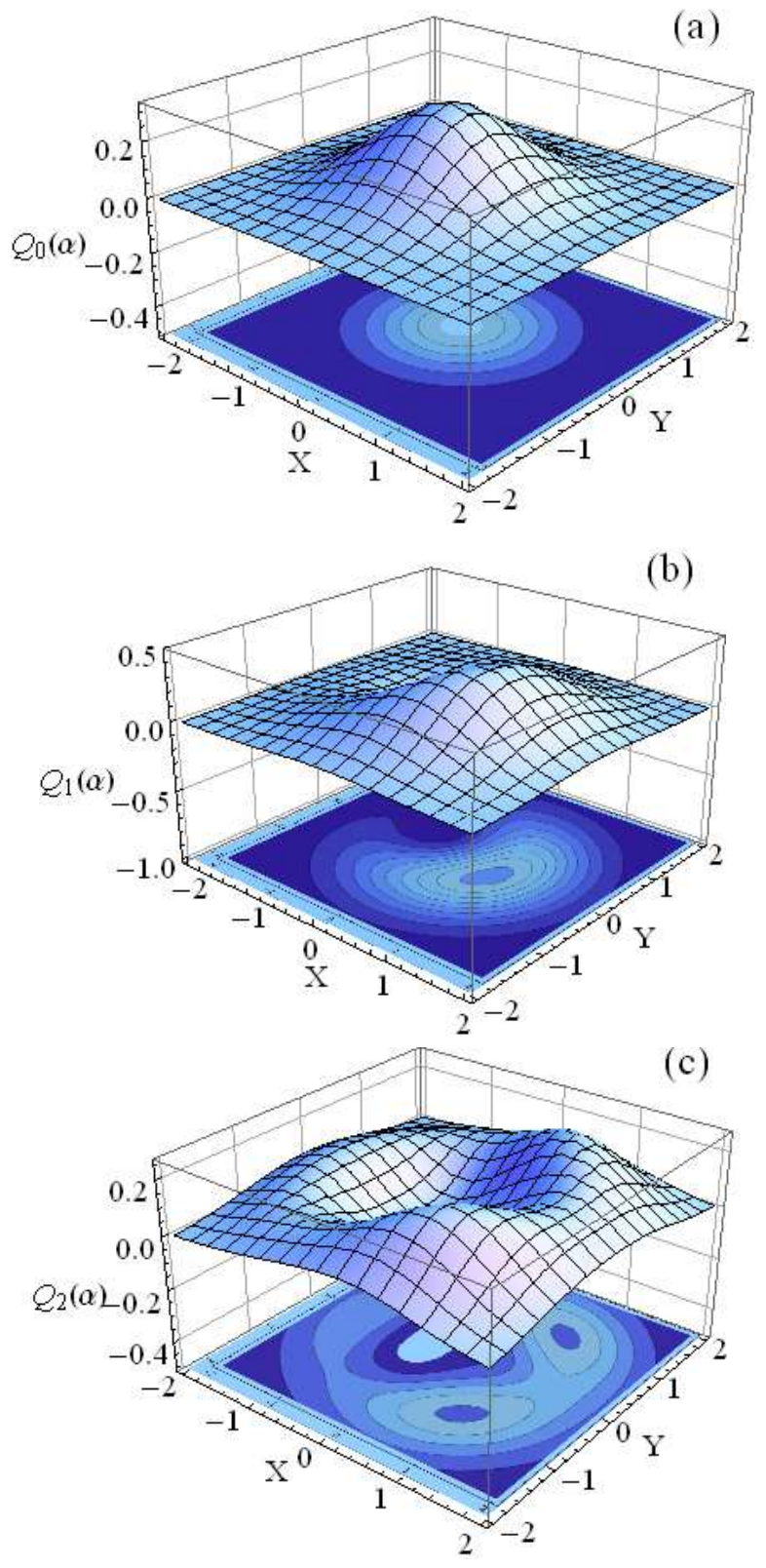

Figure 1: The $Q$-function $Q_{k}(\alpha)=\frac{\left|\left\langle\alpha \mid \varphi^{(k)}\right\rangle\right|^{2}}{\pi}$ and the counter lines of the $Q$-function for the cavity state $\left|\varphi^{(k)}\right\rangle$ after the $k^{\text {'th }}$ atom has passed through the resonator and has been detected in ground state for a fix interaction parameter $g \tau=\frac{\pi}{5}$ and (a) $k=0$, (b) $k=1$ and (c) $k=2$ (the three steps for generation of the FLCSs with $N=2$ ). 


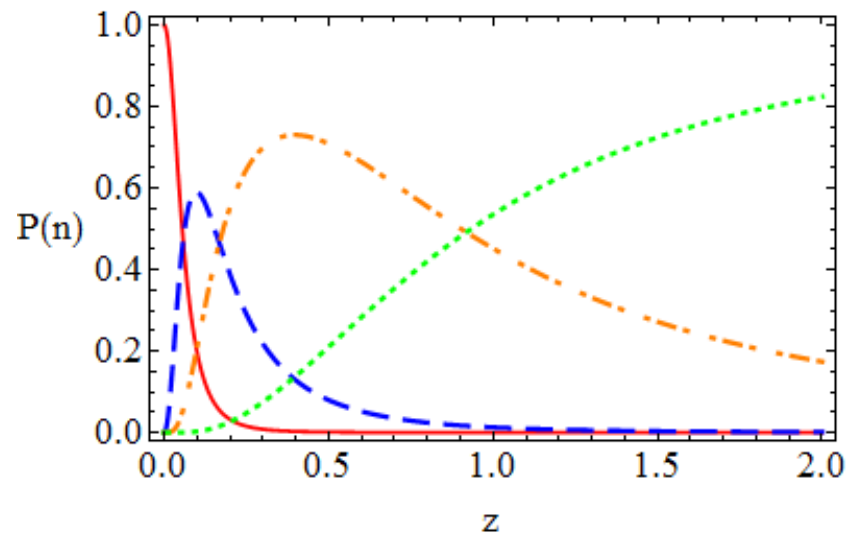

Figure 2: Probability of finding $n$ photons in the FLNCSs, versus $z$, for $\mathrm{N}=3, A=2 / 3$ and $B=1 / 3$. Here, the solid red, dashed blue, dashed-dotted orange and dotted green lines correspond to $P(0), P(1), P(2)$ and $P(3)$, respectively.

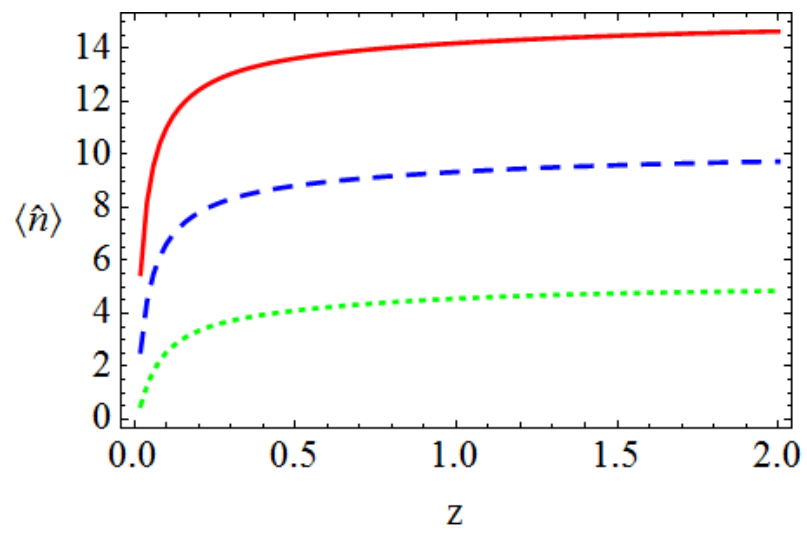

Figure 3: The mean number of photons in FLNCSs versus $z$ for $A=2 / 3$ and $B=1 / 3$. Here, the dotted, dashed and solid lines correspond to $N=5, N=10$ and $N=15$, respectively. 


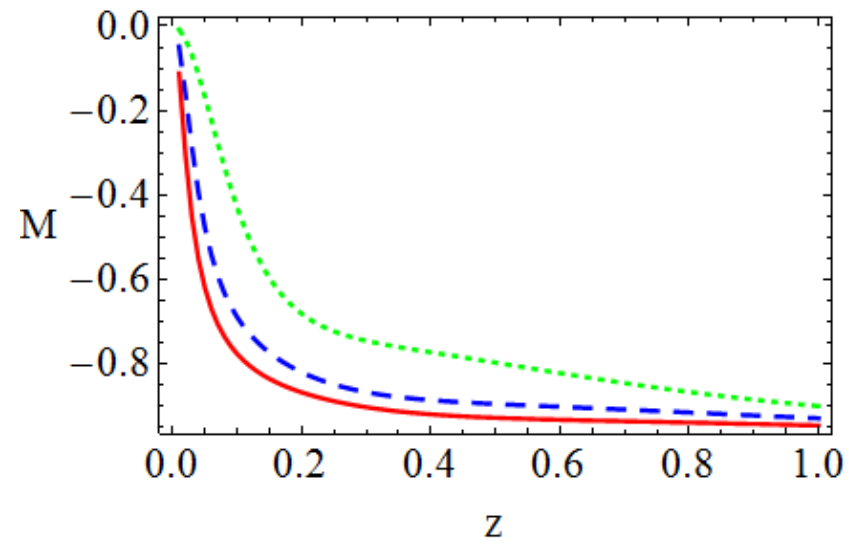

Figure 4: Mandel parameter of the FLNCSs versus $z$ for $A=2 / 3$ and $B=1 / 3$. Here, the dotted green line corresponds to $\mathrm{N}=2$, the dashed blue line to $\mathrm{N}=4$ and the solid red line to $\mathrm{N}=6$. 

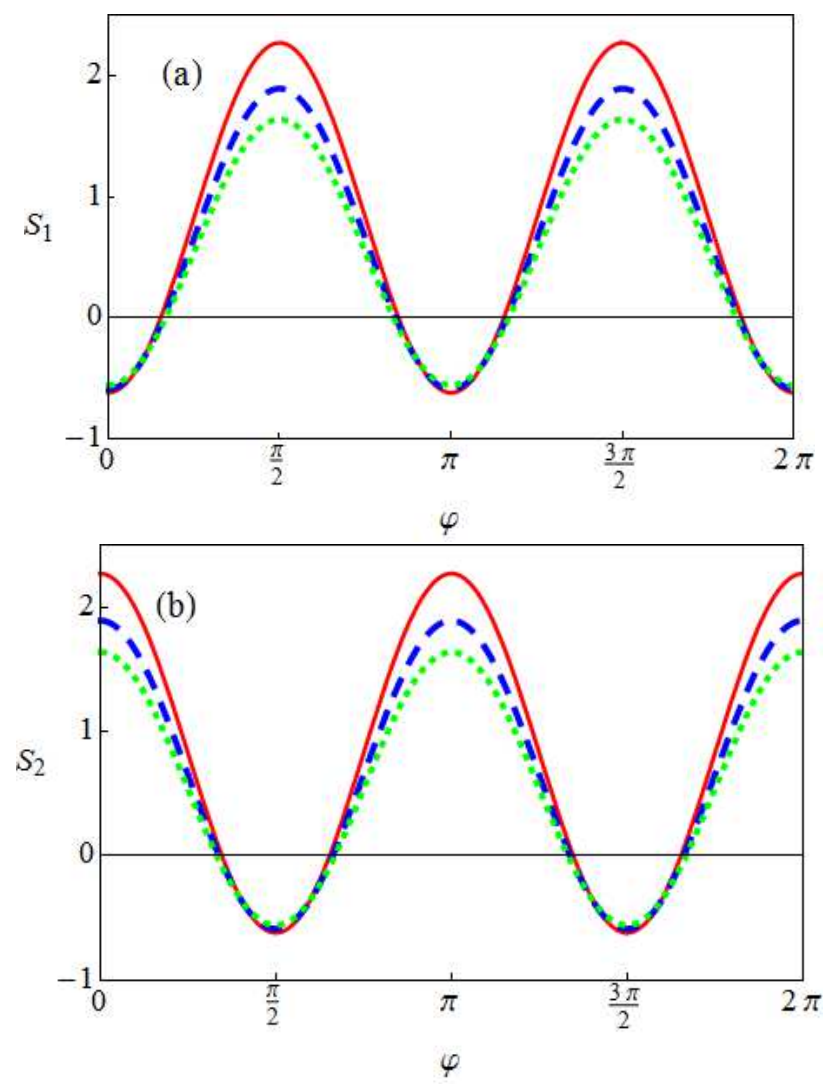

Figure 5: The squeezing parameters (a) $S_{1}$ and (b) $S_{2}$ versus $\varphi$ for $z=0.02$ and $(A, B)=$ $(2 / 3,1 / 3)$. Here, the solid-red, dashed-blue and dotted-green lines correspond to $N=20$, $N=17$ and $N=15$, respectively. 